\title{
Facile preparation of new polystyrene leucomalachite green thick films and study of their radiochromic behavior for low doses of gamma radiation
}

\author{
Anuradha Bera *, Shatrughan Malav, Bajrang Lal Tiwari, Shyam Govind Vaijapurkar \\ Radiation Dosimetry and Processing Group, Defence Laboratory, Jodhpur, India \\ *Corresponding author E-mail:anuradha_santra@yahoo.co.in
}

\begin{abstract}
Colourless polystyrene-leucomalachite green (PS-LMG) thick films containing a suitable chloroalkane were prepared by a fast and facile casting method, and were investigated for their radio chromic response behavior under the influence of $1.25 \mathrm{MeV} \gamma$-radiation. Their gamma response was studied in the $0.05 \mathrm{kGy}$ to $10 \mathrm{kGy}$ range to evaluate their suitability for potential use as the dosimeter in the radiation processing industries. The films were found to undergo a visibly distinct green coloration in the studied range, with the colour intensity increasing with an increase in the total dose. The radiochromic response of these films when investigated as a function of film thickness showed that the colour development as well as the linearity of the response was markedly affected by the thickness of the films. The effect of dye loading and the chloroalkane concentration on the radiochromic response of these films were also investigated. Depending upon the film thickness and reactant concentrations, the films were found to be capable of visually detecting gamma radiation doses as low as few tens of grays.
\end{abstract}

Keywords: Polystyrene; Leucomalachite Green, Radiochromic; Thick Films; Low Dose.

\section{Introduction}

A diverse range of radiochromic films employing $\mathrm{pH}$-sensitive dyes, leuco dyes and tetrazolium salts have been prepared and investigated for their dosimetric properties for radiation processing applications (McLaughlin et al.1989, Miller et al.1988, Mai et al.2004, Moussa et al.2003). However, most of the dosimeters prepared so far for the radiation processing industry are for monitoring medium and doses of radiation, i.e., $1 \mathrm{kGy}$ and higher ranges (Gafar et al.2015, Akhavan et al.2002, Sidney et al.1990). Recently, increase in the processes of treatment of food by radiation has realized the need for routine dosimeters and labels that requires process monitoring at relatively lower doses of radiation. Some of the food irradiation applications (Saylor et al.1988) like sprout inhibition, insect and parasite disinfection, and delay in maturation require dosimetric systems for monitoring at doses lower than $1 \mathrm{kGy}$ (Pourbeigi et al.2005). Very few (McLaughlin et al.1989, Pourbeigi et al.2005) radiochromic films have been reported for such types of low dose applications. Gafchromic films originally developed for medical dosimetry have been used in the radiation processing industries for low dose monitoring, but their routine and extensive use is often restricted by their high cost and availability.

A number of leuco based films have been prepared and investigated for their radio chromic behavior. Out of them, malachite green (MG) and its derivatives has been well studied by previous researchers for the preparation of dosimetric films as well as for 3-D dosimetry. While the polyvinyl chloride (PVC) film with malachite green was studied for high-dose radiation measurements (Kattan et al.2007, Abdel-Fattah et al.1996), polyvinyl butyral film (PVB) based on leucomalachite green (LMG) was investigated as a high-dose dosimeter for gamma radiation processing applications in the dose range of 3-150 kGy (Soliman et al.2014) while a chlorine-containing polymer matrix doped with malachite-green methoxide thin film dosimeter was reported (Akhavan et al.2007) for the dose range of 1-60 kGy. Subsequently, LMG based polyvinyl butyral as low dose dosimeters could be developed for dose measurements of the order of a few hundred Gy level up to $4 \mathrm{kGy}$ doses by Mai and group (Mai et al.2008). PVB with its specific advantages has been used as the polymer matrix by many researchers like Mei and coworkers, however, processibility of PVB is quite difficult, particularly its solubility, requiring the mixture of several high boiling solvents and co-solvents. The use of high boiling solvents not only requires longer time for the casted films to dry, it also restricts the thickness of the films owing to the skin affect as observed by previous researchers (Adamovics et al.2004). On the other hand, polystyrene being easily soluble in many common low boiling organic solvents can facilitate the preparation of much thicker films. Although polystyrene based radiochromic films have been reported earlier, but most of them have been used for the measurements of high radiation doses (McLaughlin et al.1979, Tariq et al.2012, Niroomand-Rad et al.1998).

The present study aims to prepare a new series of radio chromic leuco based polystyrene films for potential use as a dosimeter in radiation processing industry, that can respond to gamma radiation from few tens of Gy onwards, vital for many food-processing applications. For this, we have attempted to develop a new combination of an acid sensitive leuco dye, like LMG, a suitable haloalkane as the sensitivity enhancer and polystyrene as the polymer matrix. Since thicker films are known (Cheung et al.2001) to have 
higher radiation sensitivity, researchers worldwide have been using the multilayer films to improve the radiation sensitivity. Our objective was to prepare a novel formulation in such a way that all the ingredients are soluble in a single low boiling solvent like chloroform, so that faster drying can take place thereby facilitating the formation of thicker film. To the best of our knowledge, there is no such previous publication that has reported the preparation and study of radiochromic behavior of PS-LMG films for measuring low gamma doses starting from few tens of Gy onwards.

\section{Materials and methods}

\subsection{Materials}

Leucomalachite green was procured from Sigma Aldrich as colourless powder and used as such without any further purification. Polystyrene (Average MW 28, 0000) was procured from Sigma Aldrich while chloroform was procured from Sigma Aldrich and was purified further according to the reported method by Perin and Perin. Three different chloroalkanes selected for the present study, hexachloroethane, ethyl trichloroacetate and chloral hydrate were procured from Sigma Aldrich and was used without further purification.

The thickness of the solvent casted films was measured using a digital micrometer (Mitsubishi, Japan). The radiation exposure studies were carried out in Gamma Chamber (GC-5000). Fricke dosimeter was used for dose calibration. The optical characterization of the unirradiated and the irradiated films were carried out using an Ocean Optics UV-Vis reflectance spectrophotometer.

\subsection{Methods}

\subsubsection{Preparation of the PS-LMG film}

General process for the preparation of the PS-LMG films involves dissolving the three ingredients, i.e., polystyrene, LMG and the chloroalkane in chloroform in the required ratios as per the designed formulations, mixing them properly until a transparent solution is obtained and then casting them on a leveled base in a confined area. The leveling of the base was carried out by spirit level.

For example, in order to prepare a PS-LMG film with $1 \mathrm{phr}$ (parts per hundred parts of resin) of the dye and the chloroalkane ratio at 1:10, the first step was to weigh $1.0 \mathrm{gm}$ of polystyrene pellets and dissolve in $10 \mathrm{ml}$ of chloroform. $10 \mathrm{mg}$ of leucomalachite green was dissolved separately in $2 \mathrm{ml}$ chloroform. $100 \mathrm{mg}$ of the chloroalkane was weighed and was slowly added to the dye solution and mixed properly until a clear colorless solution is obtained. This dye solution containing the chloroalkane was then added to the polymer solution and the viscous formulation so obtained was ultrasonicated for 15 minutes. The solution was set aside for 1 hour for the bubbles to settle down, if any. The obtained clear viscous solution was poured into a flat glass plate having $10 \times 8$ $\mathrm{cm}^{2}$ confined area under dark room conditions, keeping temperature between $30-40{ }^{\circ} \mathrm{C}$ and letting it to dry for 18 hours. After drying, the films were carefully peeled off; thickness measured with a micrometer over multiple points, and then kept in black pouch for protection against light.

Similarly, all the other formulations were made wherein the PS, LMG, chloroalkane contents were varied, casted and dried to obtain their corresponding films. In the experiments where the radiation response as function of film thickness was studied, the formulations were kept the same; the casting area was varied.

\subsubsection{Radiation exposure studies}

For exposure studies of the prepared radiochromic film, strips were neatly cut from the film, each of size $2 \times 1 \mathrm{~cm}^{2}$. Each strip was irradiated in ${ }^{60} \mathrm{Co}$ gamma chamber at the doses up to $10 \mathrm{kGy}$. Dose rate at the time of irradiation was $3.0 \mathrm{kGy} / \mathrm{h}$.

\subsubsection{UV-VIS Spectrophotometric studies}

Quantification of the response behavior was carried out by wavelength scans of exposed and unexposed films obtained using a spectrophotometer. The response of the film was also studied by changing their orientations. A constant time gap of about 24 hours between exposure and evaluation of the film was maintained.

\section{Results and discussion}

Smooth, bubble free, colourless films of $10 \times 8 \mathrm{~cm}^{2}$ sizes were obtained by the casting method. Films of thickness ranging from $30 \pm 5 \mu \mathrm{m}$ to $300 \pm 10 \mu \mathrm{m}$ and higher were prepared by the method. Although films of thickness up to $1 \mathrm{~mm}$ could be prepared using the reported formulations and procedure, we have restricted our present study up to $300 \mu \mathrm{m}$ due to the reasons discussed in the subsequent paragraphs. While the films of $30 \pm 5 \mu \mathrm{m}$ required only 2 hours to dry, the films with $300 \pm 10 \mu$ m thickness could be obtained in 24-hour time, much lesser time period as compared to films based on other polymers like PVB, PVC and PVA. The films could be peeled off easily from the base substrate and usable as self-standing films. Another advantage of the quick drying to form these films is that the possibility of any premature coloration during the film preparation steps is minimized, resulting in completely colourless thick films.

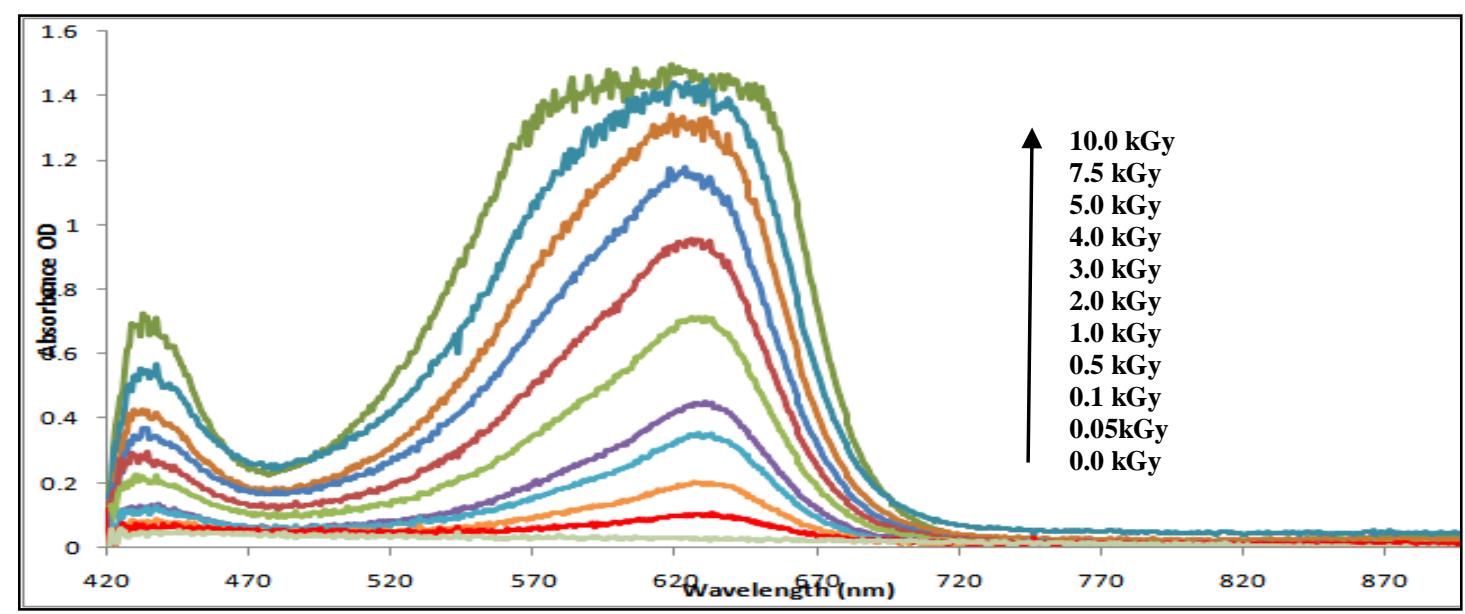

Fig. 1: Optical spectra of gamma irradiated PS-LMG film to increasing dose of radiation from $0.05 \mathrm{kGy}$ to $10 \mathrm{kGy}$. 
All the prepared films were found to respond to ${ }^{60} \mathrm{Co}-\gamma$ Radiation, the colour changes detectable visually and be easily monitored. On irradiating them with $\gamma$-radiation at regular intervals upto $10 \mathrm{kGy}$ ranges, these films became green in colour, the colour intensity increasing with increasing doses of gamma radiation. Fig. 1 shows a typical absorption curve of one of the PS-LMG films $(250 \pm 10$ micron thick with $1 \mathrm{phr}$ dye loading and 1:1 dye to hexachloroethane ratio) irradiated to increasing doses of gamma radiation upto $10 \mathrm{kGy}$.

As can be seen in the Fig.1, absorption peak at $629 \mathrm{~nm}$ wavelength was observed in the irradiated films along with another minor absorption peak at $425 \mathrm{~nm}$ that corresponded to the absorption characteristics of malachite green dye. As seen in the figure, the absorbance OD of the peak increases with an increase in radiation doses, along with the peak broadening at $629 \mathrm{~nm}$ at higher doses. No further significant increase in the Abs OD was observed beyond $10 \mathrm{kGy}$ and the peak signal almost reaching saturation at 629 $\mathrm{nm}$, although the absorbance at $425 \mathrm{~nm}$ showed a linear increase till $10 \mathrm{kGy}$. Since not much colour change was observed visibly beyond $10 \mathrm{kGy}$, it can be suggested that the colour of the radiochromic film was primarily dictated by the change in absorbance at $629 \mathrm{~nm}$.

A net change in absorbance OD at $629 \mathrm{~nm}\left(\lambda_{\max }\right)$ of the film as function of gamma doses is plotted (Fig.2). From the plot, it can be clearly seen that the change in absorbance is not linearly proportional to the radiation doses, particularly at higher doses (Fig.2). It was found from the plot that the intensity of colour increases linearly with increasing absorbed doses in the beginning of the dose-response curve then tends to increase with lower rate until reaching saturation at higher doses. The overall dose response function was found to be follow a non-linear trend $\left(2^{\text {nd }}\right.$ polynomial function); however, good linearity was observed at the low-dose ranges, almost upto $5 \mathrm{kGy}$. However, the linearity range was found to vary with a variation in the film thickness as well as in the formulation as will become evident from the subsequent studies.

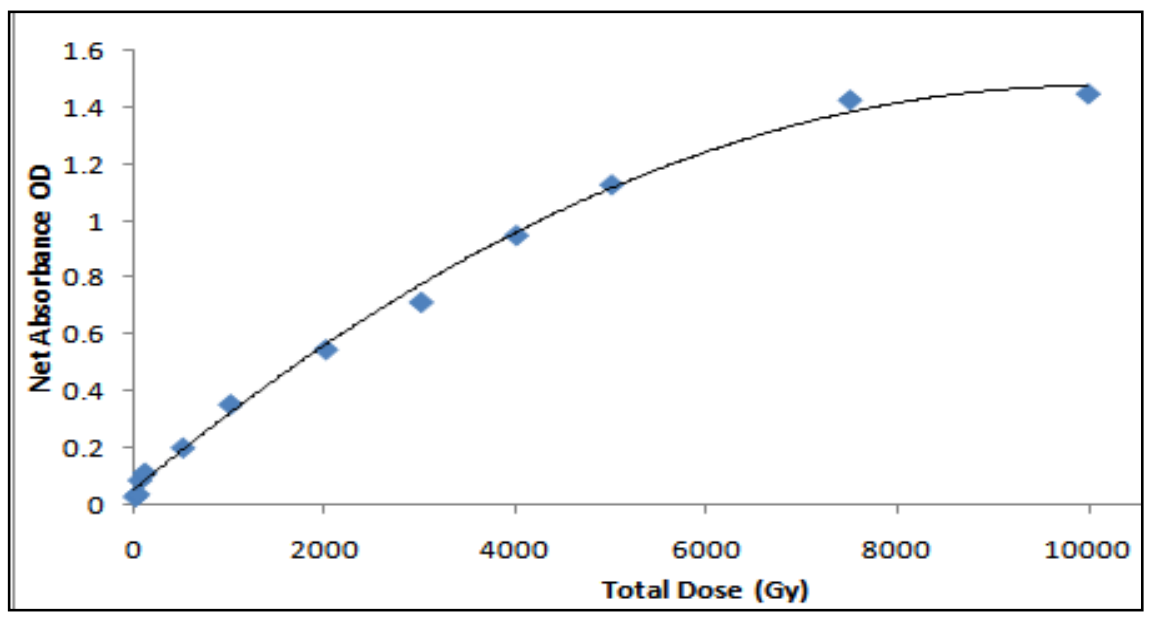

Fig.2. Plot of total dose versus net absorbance OD of the gamma irradiated PS-LMG film from $0.05 \mathrm{kGy}$ to $10 \mathrm{kGy}$

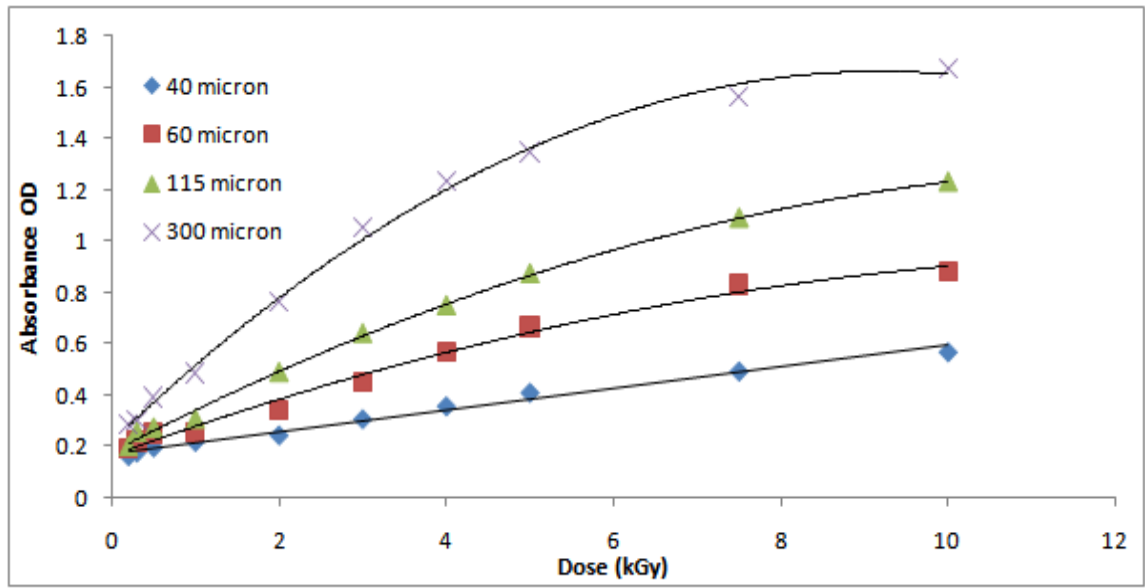

Fig. 3: Plot of Absorbance as function of increasing $\gamma$-doses up to $10 \mathrm{KGy}$ of PS-LMG films with $40 \mu \mathrm{m}, 60 \mu \mathrm{m}, 115 \mu \mathrm{m}$ and $300 \mu \mathrm{m}$ thickness. 


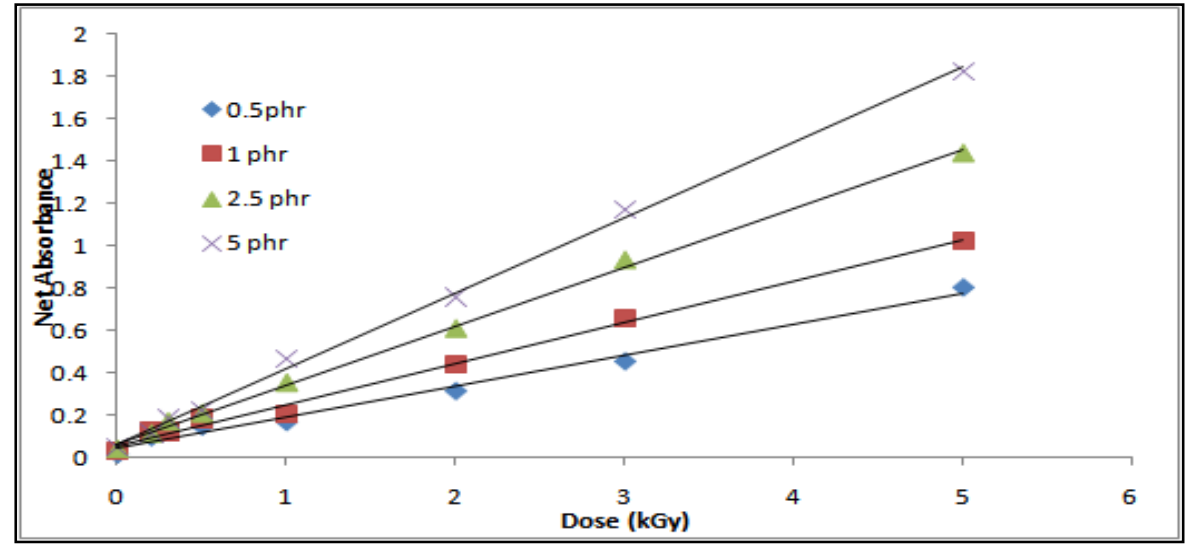

Fig. 4: Plot of Absorbance OD with increasing $\gamma$-doses upto 5kGy of LMG-PS films with 0.5phr, $1 \mathrm{phr}, 2.5 \mathrm{phr}$ and 5 Phr of LMG in the films.

In order to study the effect of film thickness on the radiochromic response of these films, films having similar composition but different thickness was irradiated with increasing doses of gamma radiation and their change in absorbance recorded. Fig. 3 shows radiation response of four such films having same composition (1 phr dye and 1:1 dye to chloroalkane ratio) but four different thicknesses, i.e., $40 \mu \mathrm{m}, 60 \mu \mathrm{m}, 115 \mu \mathrm{m}$ and $300 \mu \mathrm{m}$, and irradiated up to $10 \mathrm{kGy}$ doses, keeping all the other irradiation parameters constant. As can be seen in Fig.3, the radiochromic response of the films increase with an increase in film thickness, with the thicker films developing a more intense colour than their thinner counterparts for the same dose. For example, while a detectable colour change was observed very clearly at $50 \mathrm{~Gy}$ for a $300 \mu \mathrm{m}$ film, the $40 \mu \mathrm{m}$ film gave a detectable colour change only beyond $500 \mathrm{~Gy}$. Also the final colouration of the $300 \mu \mathrm{m}$ film after the $10 \mathrm{kGy}$ dose irradiation was much more intense than that of the $40 \mu \mathrm{m}$ film. However, the linear range for the thinner ones is found to be more than the thicker ones as clearly evident from the plot. For instance, the $300 \mu \mathrm{m}$ film shows a linear response upto $4 \mathrm{kGy}$ whereas fairly good linearity response was observed upto almost $10 \mathrm{kGy}$ for the $40 \mu \mathrm{m}$ film. Preliminary radiochromic characterization of films thicker than $300 \mu \mathrm{m}$ film, i.e., upto $1 \mathrm{~mm}$ was done in terms of their response range and visually detectable dose limit. Although the colour response was more intense, the films were found to show a very limited response range, some of them saturating even at $2 \mathrm{kGy}$, without much increase in terms of the minimum detectable dose. Detailed studies with these thicker films are in progress and will be reported subsequently, and are beyond the scope of the present publication.

The gamma sensitivity of these PS films as a function of LMG loading was studied. Films of thickness $80 \pm 5$ microns having varying dye concentration and 1:1 dye to chloroalkane ratio, was irradiated to gamma radiation doses upto $5 \mathrm{kGy}$ and their increases in colour intensity recorded as a function of increasing doses (Fig.4). The change in sensitivity of PS-LMG film with different concentration of LMG dissolved in PS matrix is shown in Fig. 4. The sensitivity of films increases proportionally to the LMG concentration which was in agreement with the observations by previous researchers (Mai et al.2008).

Since the chloroalkane concentration is known (Adamovics et al.2004) to affect the overall radiochromic response of the films, the gamma sensitivity of these PS films as a function of chloroalkane concentration was studied. We have investigated three different chloroalkanes viz., hexachloroethane, ethyl trichloroacetate and chloral hydrate. All of them were found to enhance the radiochromic response of these PS-LMG films. Fig.5. Shows the radiochromic response behavior of the thick PS-LMG films of $300 \mu \mathrm{m}$ and dyes loading $(1 \mathrm{phr})$ in presence of hexachloroethane taken in the ratio of $1: 1,1: 5,1: 10$ and $1: 25$ with respect to the LMG amount and irradiated to $\gamma$ Radiation doses upto $2 \mathrm{kGy}$. As can be seen, there is a significant influence of the dye to chloroalkane ratio on the radiochromic response behavior of these films. With higher concentration of the chloroalkane, the rate of colour change was found to increase proportionally and the

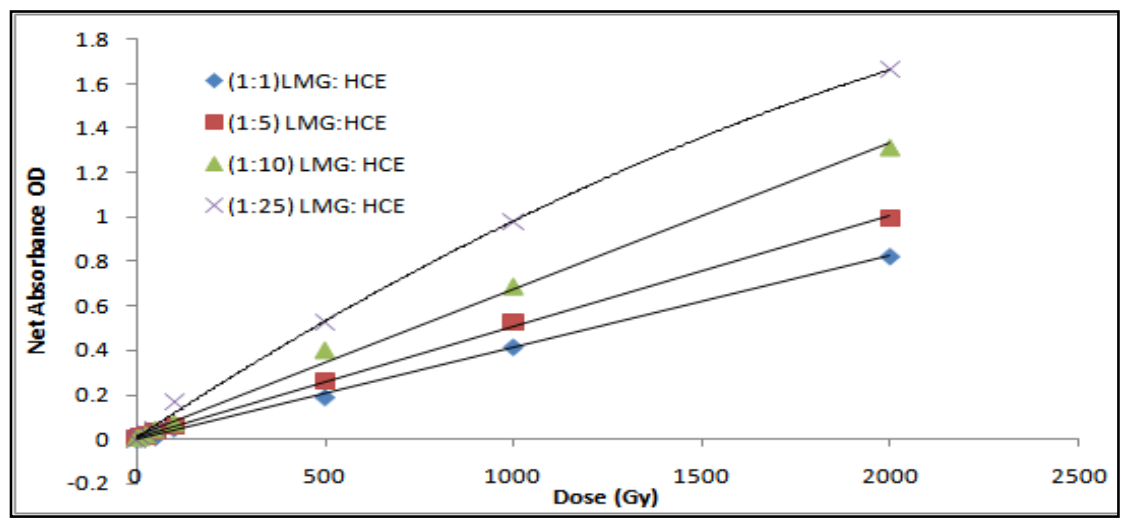

Fig. 5: Plot of Absorbance OD with increasing $\gamma$-doses upto $2 \mathrm{kGy}$ of PS-LMG films with varying ratio of LMG : Hexachloroethane ( $1: 1,1: 5,1: 10$ and $1: 25)$.

green coloration of the film becoming more intense. However, with the increase in the chloroalkane to dye ratio, the linearity range of the films is reduced, a behavioral pattern reported earlier in such kind of leuco based systems. Fig. 5 clearly shows how the linearity of the films is decreasing as we move from 1:1 to $1: 25$ dye to chloroalkane ratio.
The role of dye and chloroalkane in the radiochromic behavior of the films could well be understood from the mechanism of the colour change in the leuco based dosimeter films on interaction with ionizing radiation as investigated by several researchers previously (Abdel-Fattah et al.1996, Mai et al.1998, Whittaker et al.1987) 
The dependence of sensitivity could be explained assuming the change in both the number of colored precursors and the concentration of dye and the haloalkane in polymer matrix (Mai et al.2008). The dependence of sensitivity on the concentration of these two ingredients may be written as follows:

$$
\underline{\mathrm{D}[\text { colored dye] }}=\mathrm{k} \text { [dye precursors] } \mathrm{x} \text { [Haloalkane] }
$$

Where [colored dye] is the concentration of dye precursors to be colored, haloalkane is the active concentration of the hexachloroethane that is acting as the free radical initiator and $\mathrm{k}$ is the rate constant $\left(\mathrm{s}^{-1}\right)$

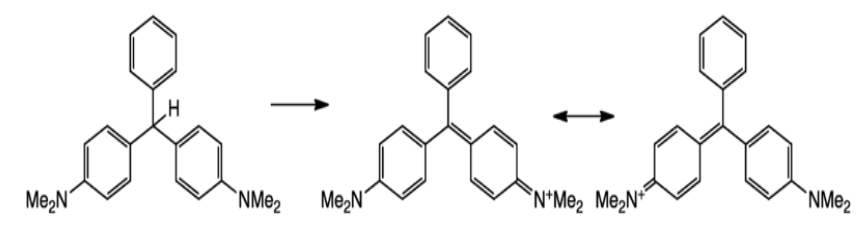

Fig. 6: Structure of LMG (Colourless) and Malachite Green (Colored)

LMG becomes coloured due to radiolytic oxidation. The RC-H bond in LMG get broken by irradiation, and it results in the structure rearrangement at one of the triarylmethane groups leading to the quinonoid chromophore structure of the deep color source as a part of the resonant unsaturated carbon ion (Fig.6) (McLaughlin et al.1977). The mechanism of the radiation induced color change of the LMG from colorless to green may be explained as similar to that of malachite green cyanide which has similar optical absorption spectrum (McLaughlin et al.1977).

The color development of the exposed portions in a radiochromic film is not detectable unless there is a radical initiator like a haloalkane in the formulation that produces radicals during the irradiation. A wide range of chloroalkanes such as chloroform, carbon tetrachloride and methylene chloride have previously demonstrated radiolytic oxidation of triphenylmethanes in liquid systems (Miyaji et al.2001) while the action of chloral hydrate have been well studied in a number of solid dosimeter films (Ebraheem et al.2013, El-Kelany et al.2012). Although not much details on the radiation induced decomposition of hexachloroethane is reported, it can be hypothesized that its radiolysis leads to the formation of chloride radicals as one of the intermediates, which in turn oxidizes the LMG and color change of the film takes place. The following mechanism for the radiolytic decomposition of hexachloroethane is proposed:

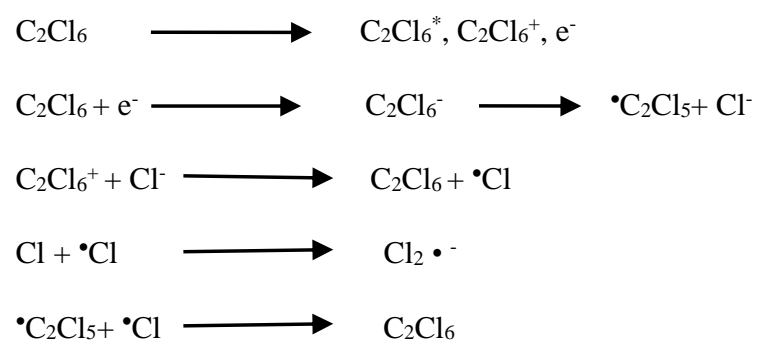

Hexachloroethane provided oxidative species $\mathrm{CI} \bullet$ and $\mathrm{Cl}_{2} \bullet-$ during irradiation, and then LMG was oxidized:

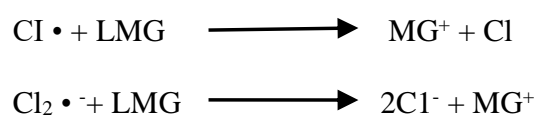

The high sensitivity of the developed leuco films may be attributed to high rate of oxidation of the leuco dye to its coloured form which in turn is probably due to the availability of large active concentration of the oxidative species $\mathrm{CI}^{*}$ and $\mathrm{Cl}_{2}{ }^{-}$formed in theradiolytic decomposition of hexachloroethane.

Therefore, it can be seen from the above observations that the thick films of PS-LMG prepared and reported here were highly sensitive towards lower dose ranges of gamma radiation. In order to establish these prepared PS-LMG films for use as dosimeters in the radiation processing industries, further studies are in progress to evaluate the effect of irradiation dose rate, temperature, humidity along with their pre- and post-irradiation stability; and will be reported in subsequent publications after the completion of the study. As mentioned earlier, the present work is mainly focused on the noble strategy adopted in terms of its preparative route and designing of formulation for the development of suitable radiochromic films that can respond to low doses of gamma radiation i.e., lesser than $1 \mathrm{kGy}$.

\section{Conclusions}

A new series of polystyrene based dosimeter films were prepared that were sensitive to low doses of gamma radiation in the 0.05 $10 \mathrm{kGy}$ range, with a minimum visually detectable dose in few tens of grays. This polystyrene based film incorporating leucomalachite green (LMG) and a chloroalkane like hexachloroethane with their thickness ranging from 30 to 300 microns was prepared using a fast and facile solvent casting method in a low boiling organic solvent like chloroform. The radiochromic response of the films, i.e., their rate of colour development and linearity range was found to be dependent on the film thickness, LMG loading as well as the concentration of chloroalkane used. Depending upon a particular radiation processing application, the radiation sensitivity of these films can be tuned by tailoring these three parameters. These films can find potential applications both as a dosimeter as well as indicator films for the food irradiation and other radiation processing industries. While a thicker PS-LMG film with higher dye loading and a higher dye to chloroalkane ratio can find its use as a colour indicator and as a low dose dosimeter for measuring doses from few tens of grays, the thinner films with lesser content of dye and lower dye to chloroalkane ratio will be more suitable for dose measurements in the relatively higher ranges, ie., from few hundreds of grays upto more than $10 \mathrm{kGy}$ of gamma doses.

\section{Acknowledgement}

The authors are extremely grateful to Dr. S.R. Vadera, Director, Defence Laboratory, and Jodhpur for his constant help and encouragement in carrying out the work. The authors are also thankful to Sh. G.L. Baheti, Divisional Head, NRMA Division for his kind support. The authors acknowledge the help and cooperation of the staff of RAVI.

\section{References}

[1] Gafar SM \& El-Ahdal MA, (2015) A new developed radiochromic film for high dose dosimetry applications. Dyes and pigments, Elsevier, vol. 114.

[2] Soliman YS, Basfar AA \& Msalam RI (2014) A radiochromic film based on leucomalachite green for high dose dosimetry application. Radiation measurements, 62, 45-51. https://doi.org/10.1016/ j.radmeas.2014.01.004

[3] Ebraheem S \& El-Kelany M (2013) Dosimeter Film Based on Ethyl Violet-Bromophenol Blue Dyed Poly (Vinyl Alcohol). Open Journal of Polymer Chemistry, 3, 1-5. https://doi.org/10.4236/ ojpchem.2013.31001.

[4] El-Kelany M, Ebraheem S \& Gafar SM (2012) Development of a plastic dosimeter for industrial use with high doses. Chemistry and Materials Research 2, 39-46.

[5] Tariq J Alwan (2012) Gamma irradiation effect on the optical properties and refractive index dispersion of dye doped polystyrene films. Turk J Phys, 36, 377-384.

[6] Mai HH, Solomon HM, Taguchi M \& Kojima T (2008) Polyvinyl butyral films containing leuco-malachite green as a low-dose do- 
simeter. Radiat. Phys. and Chem., 77, 457-462. https:// doi.org/10.1016/j.radphyschem.2007.06.012.

[7] Akhavan A, Sohrabpour M \& Sharifzadeh M (2007) Dosimetric Evaluation of a Newly Developed Radiochromic Film for Radiation Processing. Iranian Journal of Science \& Technology, Transaction A, 31, 397-401.

[8] Kattan M, Daher Y \& Alkassiri H (2007) A high-dose dosimeterbased polyvinyl chloride dyed with malachite green. Radiat Phys Chem; 76: 1195-99. $\quad$ https://doi.org/10.1016 /j.radphyschem.2006.12.004

[9] Pourbeigi H, Meigooni AS, Ghafourian H, Koona RA \& Zahmatkesh MH (2005) Enhancement of MD-55-2 radiochromic film sensitivity using a multilayer film technique for applications in the low dose range. Iran. J. Radiat. Res., 3 (1): 11-15.

[10] Adamovics J \& Maryanski MJ (2004) A new approach to radiochromic three-dimensional dosimetry-polyurethane. Journal of Physics: $\quad$ Conference $\quad$ Series 3 172-175. https://doi.org/10.1088/1742-6596/3/1/020.

[11] Mai HH, Duong ND \&Kojima T (2004) Dyed polyvinylchloride films for use as high-dose routine dosimeter for radiation processing.Radiat. Phys. Chem. 69, 439-444. https://doi.org/ 10.1016/j.radphyschem.2003.08.006.

[12] Moussa A, Baranyai, Wojnárovits, ML, Kovács A \& McLaughlin WL (2003) Dosimetry characteristics of the nitro blue tetrazolium polyvinylalcohol film for high dose applications. Radiat. Phys. Chem., 68, 1011-1015. https://doi.org/10.1016/j.radphyschem. 2003.09.003.

[13] Akhavan A, Sohrabpour M \& Sharifzadeh M (2002) Preparation of a new chemical radiochromic film dosimeter. Radiat. Phys. Chem. 63, 773-775. https://doi.org/10.1016/S0969-806X(01)00610-7.

[14] Cheung T, Buston MJ \& Yu RK (2001) Use of multiple layers of Gafchromic film to increase sensitivity. Phys Med Biol, 46, 235240. https://doi.org/10.1088/0031-9155/46/10/401.

[15] Miyaji T, Tokita T \& Tachikawa T (2001) Development of a Radiation Dosimeter Consisting of Leuco Crystal Violet and a Small Amount of Halocarbons. Journal of Photopolymer Science and Technology, 14, 225-226. https://doi.org/10.2494/ photopolymer.14.225.

[16] Abdel-Fattah AA, \& El-Kelany M (1998) Radiation sensitive Indicator based on Radiation-Chemical Formation of Acids in Polyvinyl Butyal Films Containing Chloral Hydrate. Radiat. Phys. Chem. 51, 317-325. https://doi.org/10.1016/S0969-806X(97)00258-2.

[17] Niroomand-Rad A, Blackwell CR, Coursey BM, Gall KP, Galvin JM, McLaughlin WL, Meigooni AS, Nath R, Rodgers JE \& Soares CG (1998) Radiochromic Film Dosimetry Recommendations of AAPM Radiation Therapy Committee Task Group No. 55. Med Phys 25, 2093-2115. https://doi.org/10.1118/1.598407.

[18] Abdel-Fattah AA, Ebraheem S, El-Kelany M \& Abdel Rehim (1996) High dose film dosimeters based on Bromophenol Blue or Xylenol Orange dyed polyvinyl alcohol. Appl. Radiat. Isot. 47:345350.https://doi.org/10.1016/0969-8043(95)00266-9.

[19] Sidney LN, Lynch DC \& Willet PS (1990) A new radiochromic dosimeter film. Radiat. Phys. Chem. 35 , 770.https://doi.org/10.1016/1359-0197(90)90315-9.

[20] McLaughlin WL, Boyd AW, Chadwick KH, McDonald JC \& Miller A (1989) Dosimetry for radiation processing. London. Taylor \& Francis ISBN 0-85066-740-2.

[21] Miller A, Batsberg W \& Karman W (1988) A new radiochromic thin film dosimeter system. Radiat. Phys.Chem. 31, 491-496.

[22] Saylor MC, Tamargo TT, Mclaughlin WL, Khan HM, Lewis DF \& Schenfele RD (1988) A thin film recording medium for use in food Irradiation. Radiat. Phys. Chem. 31, 529-536. https://doi.org/10.1016/1359-0197(88)90222-6.

[23] Whittaker, B (1987) Radiation-sensitive material, UK. Patent Application GB 2182941 A.

[24] McLaughlin WL, Humphreys JC, Radak BB, Miller A \& Olejnik TA (1979) The response of plastic dosimeters to gamma rays and electrons at high absorbed dose rates. Radiat. Phys. Chem. 14 535-550. https://doi.org/10.1016/0146-5724(79)90090-6.

[25] McLaughlin WL, Miller A, Fidan S, Pejtersen K, \& Pederson WB (1977) Radiochromic plastic films for accurate measurement of radiation absorbed dose and dose distributions. Radiat. Phys. Chem. 10, 119-127. https://doi.org/10.1016/0146-5724(77)90017-6. 